\title{
Attitude and Practice Towards Exclusive Breast Feeding and Its Associated Factors Among HIV Positive Mothers in Southern Ethiopia
}

\author{
Kassa Eshetu Modjo ${ }^{1}$, Negash Wakgari Amanta ${ }^{2, *}$ \\ ${ }^{1}$ Southern Nation, Nationalities and People's regional Health Bureau, Hawassa, Ethiopia \\ ${ }^{2}$ School of Nursing and Midwifery, College of Medicine and Health Sciences, Hawassa University, Hawassa, Ethiopia
}

Email address:

kassaeshetum@yahoo.com (K. Eshetu), negashwakgari@yahoo.com (N. Wakgari)

\section{To cite this article:}

Kassa Eshetu Modjo, Negash Wakgari Amanta. Attitude and Practice Towards Exclusive Breast Feeding and Its Associated Factors Among HIV Positive Mothers in Southern Ethiopia. American Journal of Health Research. Vol. 3, No. 2, 2015, pp. 105-115.

doi: 10.11648/j.ajhr.20150302.18

\begin{abstract}
Introduction: The controversy between the risk of HIV transmission through breast milk and the life saving benefits of breastfeeding remains to be a dilemma faced by HIV positive mothers. Cognizant of this, World Health Organization recommends avoidance of all breastfeeding by HIV positive mothers when replacement feeding is acceptable, feasible, affordable, sustainable and safe. Otherwise, exclusive breast feeding is recommended during the first 6 months of life. Despite this effort, exclusive breast feeding is uncommon practice among HIV infected women. The aim of this study was to assess attitude and practice towards exclusive breast feeding and its associated factors among HIV positive mothers attending public hospitals of southern Ethiopia, 2013. Methods: Institution based cross-sectional study was conducted from August 1 to September 15, 2013. A total of 436 HIV positive mothers were recruited in the study. Pretested and structured interviewer administered questionnaires were used for data collection. Data were entered to EpiInfo and exported to SPSS for further analysis. Logistic regression analyses were used to see the association of different variables. Odds ratios and $95 \%$ confidence interval were computed to determine the presence and strength of association. Results: More than half (56.7\%) of mothers had favorable attitude towards exclusive breast feeding and nearly half (48.2\%) mothers exclusively breast feed their infants. Mother's education, occupation, house hold income, antenatal care attendance, counseling and HIV disclosure to spouse/family members were found to be associated with attitude and practice towards exclusive breast feeding. Conclusion: The attitude and prevalence of exclusive breast feeding practice were found to be very low. Therefore, HIV positive women opting to breastfeed should be encouraged to exclusively breast feed their infants and need to be promoted by policy makers and implementers.
\end{abstract}

Keywords: Exclusive Breast Feeding, Ethiopia, HIV Positive Mothers

\section{Introduction}

Exclusive breast-feeding (EBF) is defined as the consumption of only breast milk with no supplementation of any type since birth except drops and syrups like; vitamins, minerals or medicines. Breastfeeding is a major health promoting factor for infants and children in developing countries but the risk of mother-to-child transmission (MTCT) of human immune virus (HIV) by this route is challenging traditional practices $[1,2]$.

Globally, an estimated 2.1 million of children under 15 years of age are living with human immune virus or Acquired Immune Deficiency Syndrome (HIV/AIDS). Of these children $90 \%$ are living in sub-Saharan Africa, while about
64,813 are living in Ethiopia. In the absence of intervention, $30-45 \%$ of infants born to HIV-positive mothers in developing countries become infected during pregnancy, child birth and breastfeeding [3-5]. World Health Organization (WHO) and United Nations Children's Emergency Fund (UNICEF) recognized that breast feeding contributed for about 300,000 HIV infections per year worldwide, while at the same time 1.5 million children die each year if the women do not to breastfeed. The vast majority of these infections and deaths occur in Sub-Saharan Africa [6]. The health and survival benefits of breastfeeding exceed the risks of HIV transmission. However, the controversy between the risk of HIV transmission through breast milk and the life saving benefits of breastfeeding 
creates an impossible dilemma among HIV positive mothers in developing countries. WHO recommends avoidance of all breast feeding by HIV infected mothers when replacement feeding is acceptable, feasible, affordable, sustainable, and safe (AFASS). Otherwise, EBF is recommended during the first 6 months of life [7]. The EBF practice is special for many reasons, including: bonding between mothers and baby, cost saving, perfect nutrition and health benefits for baby [8]. In spite of this fact, providing lifelong antiretroviral drugs to all pregnant women is the most effective way to prevent mother-to-child transmission(test and treat protocol) [7].

EBF is the single most effective intervention to save the lives of infants and children in developing countries. Approximately, $13-15 \%$ of deaths of children aged less than 5 years could be prevented if universal coverage of EBF were increased to $90 \%$ among infants aged less than 6months [9]. Despite strong evidence that supports EBF, its prevalence has still remained very low worldwide (39\%), and it is estimated to be $47 \%$ in Africa. Its success in practice has also been very low in Ethiopia [10-13]. Although data on attitude of EBF is limited, many studies have been employed on EBF practice in Africa, while only little information is available in Ethiopia. Therefore, this study was attempted to assess attitude and practice towards exclusive breast feeding and its associated factors among HIV positive mothers attending public hospitals of southern Ethiopia.

\section{Methods}

\subsection{Study Design and Set Up}

Institution based cross-sectional study design was conducted from August 1, 2013 to September 14, 2013 among HIV positive mothers attending prevention of mothers to child transmission (PMTCT) and anti-retroviral therapy (ART) clinics in selected public hospitals of Southern Nation, Nationalities and People's Regional State (SNNPRS), southern Ethiopia. SNNPRS is the third largest administrative region of Ethiopia. Hawassa is the capital of the region, which is $275 \mathrm{kms}$ away from Addis Ababa. Administratively the state is divided into 15 Zones and 4 special woredas. It represents about $20 \%$ of the country's population with a projected population size of $17,857,192$. Of these, 9,107,168 are females. Women of child bearing age (15-49) are estimated to be $2,121,970$. Of which $3.9 \%$ are pregnant. It has 22 (18 governmental and 4 nongovernmental) hospitals, 563 health centers and 3,535 health posts during the study. According to the 2007 report of Antenatal care Sentinel HIV Surveillance in Ethiopia, HIV prevalence of pregnant women in this region is $2.1 \%$ [14].

\subsection{Sample Size and Sampling Procedure}

The sample size was calculated using single population proportion formula: $\mathrm{n}_{\mathrm{o}}=(\mathrm{Z} \alpha / 2)^{2} * \mathrm{p}(1-\mathrm{p}) / \mathrm{w}^{2}$, by considering the following assumptions: the proportion $(p)=16.2 \%$, awareness about feeding options among HIV positive mothers in Guraghe Zone [15]. 95\% confidence level of
$\mathrm{Z} \alpha / 2=1.96,5 \%$ of absolute precision and 5\% non-response rate. Design effect 2 was applied. Hence, the total sample size was 439. All HIV positive mothers who had infants/last child aged $\leq 2$ years attending PMTCT and ART clinics in public hospitals of SNNPRS were considered as the source population.

Multi-stage sampling technique was applied: Among 18 governmental hospitals in the state, nine of them were taken by simple random sampling techniques using lottery method. The sample size was distributed to each PMTCT/ART clinics of each selected hospitals using probability proportional to size (PPS) and the numbers of HIV positive mothers required for the study in each PMTCT/ART clinics were determined. Sample size for each PMTCT/ART sites was computed as follows using the formula: $\mathrm{ni}=\mathrm{Nixno} / \mathrm{N}$, Where, $\mathrm{ni}=$ number of HIV positive mothers that are needed for the study in each PMTCT/ART clinic/hospital. $\mathrm{Ni}=$ total number of $\mathrm{HIV}$ positive mothers who are attending the service at each PMTCT/ ART clinic/hospital, no=calculated sample size and "N" is total population of all selected hospitals in the region.

Therefore, each study subject was sampled from respective hospitals by systematic random sampling techniques and a total of 436 study subjects attending PMTCT and ART clinics were included in the study. The study population was listed and arranged in order. Sampling intervals were determined by dividing $\mathrm{N} / \mathrm{n}(1,565 / 439=4)$, i.e. every $4^{\text {th }}$ intervals and the starting point was chosen randomly.

\subsection{Data Collection Tools and Procedure}

Pretested and structured questionnaires using face-to-face interviewing with HIV positive mothers were used for data collection. Different relevant literatures were reviewed to develop the tool that addresses the objective of the study $[1,4$, 6,10-11,15-16]. Pre-testing of the questionnaire was undertaken on $30 \mathrm{HIV}$ positive mothers at non sampled hospitals. Findings from the pretest were used to modify the instrument. The questionnaire was designed to obtain information on the sociodemographic and obstetric characteristics of mothers, knowledge about EBF, and their attitude towards EBF practice. Data were collected by 13 diploma nurses and 5 diploma midwives (supervised by 3 B.Sc nurses and 3 health officers). A two days comprehensive training was given to data collectors and supervisors. The questionnaire was first prepared in English and then translated into Amharic (the local language), and back into English to ensure consistency.

\subsection{Measurements}

Attitude towards EBF was measured using 5 points Likert scale. The order of scoring for negative statements was reversed (strongly disagree $=5$, disagree $=4$, undecided $=3$, agree $=2$, agree $=1$ ) and the reverse was true for positive statements. The total score was obtained and computed for mean in order to categorize in to favorable and unfavorable attitudes. Score $\geq$ mean was taken as favorable attitude, while that score $<$ mean was taken as unfavorable attitude. 
Exclusive breast feeding practice is a current use, whether the respondents reported current child feeding practices.

Knowledge about EBF was measured by using ten knowledge questions. If respondents answered correctly for six or more questions, it was taken as good knowledge otherwise, poor knowledge for those who answered correctly for less than six questions.

\section{Data Processing and Analysis}

The collected questionnaire was checked manually for its completeness, coded and entered into EpiInfo version 3.5.1 statistical package, then exported to SPSS version 20.0 for further analysis. Descriptive and summary statistics were done. Both bivariate and multivariate logistic regression analysis was used to determine the association of each independent variable with the dependent variable. Variables significant in bi-variate analysis were entered into a multivariate logistic regression model to adjust the effects of cofounders on the outcome variable. Odds ratio with their $95 \%$ confidence intervals were computed to identify the presence and strength of association, and statistical significance was declared if $\mathrm{p}<0.05$.

\section{Ethical Considerations}

Ethical approval was obtained from the Institutional Review Board of the University of Gondar and submitted to SNNPRS Health Bureau. Permission letter was granted from SNNPRS Health Bureau to respective health institutions. Verbal consent was obtained from each study subject prior to data collection process preceded. Those respondents who were not willing to participate in the study were not forced to involve. Their privacy was maintained. To keep their confidentiality personal identifiers were not utilized.

\section{Results}

\subsection{Socio-Demographic Characteristics}

A total of 436 mothers were included in the study. The overall response rate was $99.3 \%$. Of these mothers $324(74.3 \%)$ were aged between $25-34$ years. Sixty one (14.0\%) mothers were aged $\geq 35$ years, while $51(11.7 \%)$ of them were aged between 15-24 years old. The mean age of the respondents was 29(SD 4.4) years old. Among infants/children of these mothers, $257(58.9 \%)$ were aged between 12-24 months. The mean age of infants/children was 12.5 (SD 5.6) months old. Of these infants/children, 234(53.7\%) were male. The majority, 349(80.0\%) mothers were married, $31(7.1 \%)$ mothers were widowed, while $26(6.0 \%), 23(5.3 \%)$ and $7(1.6 \%)$ of them were divorced, single and separated respectively. By religious affiliation, 194(44.5\%) mothers were Orthodox Christians, and 178(40.8\%) mothers were Muslim, while Protestant accounts for, 59(13.5\%). By ethnic composition, 107(24.5\%) mothers were Wolayita, 62(14.2\%) mothers were Sidama, 95 (21.7\%) mothers were Amhara, 51(11.7\%) mothers were Oromo, $43(9.9 \%)$ were Guraghe and similar proportions were Gedeo and $11(2.5 \%)$ mothers were Hadiya, while the rest were categorized in others (Kaffa and Siltie) (Table 1).

Table 1. Socio-demographic characteristics, SNNPR Hospitals, August, 2013 .

\begin{tabular}{|c|c|c|}
\hline Variables $[n=436]$ & Frequency & Percent \\
\hline \multicolumn{3}{|c|}{ Educational status of mother } \\
\hline Cannot read and write & 124 & 28.4 \\
\hline Only read and write & 96 & 22.0 \\
\hline Primary education & 121 & 27.8 \\
\hline Secondary education & 67 & 15.4 \\
\hline Higher education & 28 & 6.4 \\
\hline \multicolumn{3}{|c|}{ Educational status of spouse } \\
\hline Cannot read \& write & 33 & 8.0 \\
\hline Only read and write & 87 & 21.0 \\
\hline Primary education & 91 & 21.9 \\
\hline Secondary education & 108 & 26.0 \\
\hline Higher education & 96 & 23.1 \\
\hline \multicolumn{3}{|c|}{ Occupation of the mother } \\
\hline Housewife & 164 & 37.6 \\
\hline Gov't employee & 85 & 19.5 \\
\hline Merchant & 111 & 25.5 \\
\hline Farmer & 27 & 6.2 \\
\hline Others* & 49 & 11.2 \\
\hline \multicolumn{3}{|l|}{ Monthly income } \\
\hline$\leq 500 \mathrm{ETB}$ & 100 & 22.9 \\
\hline 501-1500 ЕТВ & 202 & 46.2 \\
\hline 1501-2500 ЕТВ & 64 & 14.6 \\
\hline$>2500$ ETB & 70 & 16.3 \\
\hline \multicolumn{3}{|l|}{ Residence } \\
\hline Urban & 369 & 84.6 \\
\hline Rural & 67 & 15.4 \\
\hline \multicolumn{3}{|l|}{ Number of children } \\
\hline One & 95 & 21.8 \\
\hline Two & 194 & 44.5 \\
\hline Three to five & 128 & 29.4 \\
\hline More than five & 19 & 4.3 \\
\hline \multicolumn{3}{|c|}{ Gender of infants/last child } \\
\hline Male & 234 & 53.7 \\
\hline Female & 202 & 46.3 \\
\hline \multicolumn{3}{|c|}{ Age of infants/last child in group } \\
\hline$<=6$ months & 72 & 16.5 \\
\hline $7-11$ months & 107 & 24.5 \\
\hline 12-24months & 257 & 59.0 \\
\hline
\end{tabular}

Others* = daily laborers \& unemployed

$\mathrm{ETB}=$ Ethiopian Birr

\subsection{Attitude Towards Exclusive Breastfeeding}

In this study, more than half $(56.7 \%)$ of mothers had favorable attitude towards EBF. A much lower percentage $(22.0 \%)$ of mothers agreed that EBF transmits HIV. Significant proportion, $(82.6 \%)$ of mothers agreed that breast feeding is nutritionally complete. In contrast, nearly half $(46.8 \%)$ of mothers agreed that formula feeding is 
nutritionally complete (Table 2 ).

Table 2. Attitude towards EBF, SNNPR Hospitals, August, 2013.

\begin{tabular}{|c|c|c|}
\hline Variables $[n=436]$ & Frequency & Percent \\
\hline \multicolumn{3}{|l|}{ Overall attitude } \\
\hline Favorable & 247 & 56.7 \\
\hline Unfavorable & 189 & 43.3 \\
\hline \multicolumn{3}{|c|}{ EBF for 6 months is the best choice for infant } \\
\hline Disagree & 88 & 20.2 \\
\hline Undecided & 9 & 2.0 \\
\hline Agree & 339 & 77.8 \\
\hline \multicolumn{3}{|c|}{ EBF is not good since it transmits HIV } \\
\hline Disagree & 298 & 68.4 \\
\hline Undecided & 42 & 9.6 \\
\hline Agree & 96 & 22.0 \\
\hline \multicolumn{3}{|c|}{ EBF for 6 months is nutritionally complete } \\
\hline Disagree & 65 & 14.9 \\
\hline Undecided & 11 & 2.5 \\
\hline Agree & 360 & 82.6 \\
\hline \multicolumn{3}{|c|}{ Formula feeding is nutritionally complete } \\
\hline Disagree & 189 & 43.3 \\
\hline Undecided & 43 & 9.9 \\
\hline Agree & 204 & 46.8 \\
\hline \multicolumn{3}{|c|}{ ERF for 6 months is best choice for infants/last child } \\
\hline Disagree & 237 & 54.4 \\
\hline Undecided & 39 & 8.9 \\
\hline Agree & 160 & 36.7 \\
\hline \multicolumn{3}{|c|}{ I do not accept ERF for fear of stigma due to HIV } \\
\hline Disagree & 308 & 70.6 \\
\hline Undecided & 42 & 9.7 \\
\hline Agree & 86 & 19.7 \\
\hline \multicolumn{3}{|c|}{ Mixed feeding has risk of HIV infection to infant/last child } \\
\hline Disagree & 129 & 29.6 \\
\hline Undecided & 40 & 9.2 \\
\hline Agree & 267 & 61.2 \\
\hline \multicolumn{3}{|c|}{ Mixed feeding is always necessary to infants/last child } \\
\hline Disagree & 214 & 49.5 \\
\hline Undecided & 63 & 14.4 \\
\hline Agree & 157 & 36.1 \\
\hline \multicolumn{3}{|c|}{ Heat treated milk is very important to my infant } \\
\hline Disagree & 119 & 27.3 \\
\hline Undecided & 77 & 17.7 \\
\hline Agree & 240 & 55.0 \\
\hline \multicolumn{3}{|c|}{ Wet-nurse is good infant feeding option } \\
\hline Disagree & 269 & 61.7 \\
\hline Undecided & 80 & 18.3 \\
\hline Agree & 87 & 20.0 \\
\hline \multicolumn{3}{|c|}{ Bottle feeding is good infant feeding option } \\
\hline Disagree & 140 & 32.1 \\
\hline Undecided & 28 & 6.4 \\
\hline Agree & 268 & 61.5 \\
\hline \multicolumn{3}{|c|}{ Bottle feeding is not good infant feeding option } \\
\hline Disagree & 259 & 59.4 \\
\hline Undecided & 26 & 6.0 \\
\hline Agree & 151 & 34.6 \\
\hline \multicolumn{3}{|c|}{ Complementary foods after 6 months is best choice } \\
\hline Disagree & 38 & 8.7 \\
\hline Undecided & 13 & 3.0 \\
\hline Agree & 385 & 88.3 \\
\hline
\end{tabular}

\subsection{Exclusive Breast Feeding Practice}

The majority, $361(82.8 \%)$ of mothers were fully breastfed their infant/last child. One hundred eighty six $(51.5 \%)$ mothers were initiated breast feeding immediately after birth. Comparatively few, $38(8.7 \%)$ mothers were offered fluids and solid foods (pre-lacteal feeding) to their infant/last child immediately after birth. Among reasons cited by respondents for providing pre-lacteal feeding were milk did not come in yet accounted for (63.2\%) (Table 3).

Table 3. EBF Practice, SNNPR Hospitals, August, 2013.

\begin{tabular}{|c|c|c|}
\hline Variables & Frequency & Percent \\
\hline \multicolumn{3}{|l|}{ Ever breast feeding practice } \\
\hline Yes & 361 & 82.8 \\
\hline No & 75 & 17.2 \\
\hline \multicolumn{3}{|l|}{ Initiation of breast feeding } \\
\hline Immediately after delivery & 186 & 51.5 \\
\hline Within 30 minutes after delivery & 62 & 17.2 \\
\hline Within 1 hour after delivery & 103 & 28.5 \\
\hline Others* & 10 & 2.8 \\
\hline \multicolumn{3}{|l|}{ Pre-lacteal feeding } \\
\hline Yes & 38 & 8.7 \\
\hline No & 398 & 91.3 \\
\hline \multicolumn{3}{|l|}{ Reasons for pre-lacteal feeding } \\
\hline Milk did not come in yet & 24 & 63.2 \\
\hline I was ill & 7 & 18.4 \\
\hline Baby was ill & 3 & 7.9 \\
\hline Others* & 4 & 10.5 \\
\hline \multicolumn{3}{|l|}{ Still breast feeding } \\
\hline Yes & 171 & 47.4 \\
\hline No & 190 & 52.6 \\
\hline \multicolumn{3}{|l|}{ Duration of Breast feeding } \\
\hline $0-6$ months & 189 & 52.4 \\
\hline 12 months & 99 & 27.4 \\
\hline 18-24 months & 73 & 20.2 \\
\hline \multicolumn{3}{|l|}{ Frequency of breast feeding } \\
\hline $2-5$ times & 47 & 13.0 \\
\hline 8-10 times & 245 & 67.9 \\
\hline Frequent and on demand & 69 & 19.1 \\
\hline \multicolumn{3}{|l|}{ Exclusive breast feeding practice } \\
\hline Yes & 210 & 48.2 \\
\hline No & 226 & 51.8 \\
\hline \multicolumn{3}{|l|}{ Duration of Exclusive Breastfeeding } \\
\hline $1-2$ months & 3 & 1.4 \\
\hline 3 months & 4 & 1.9 \\
\hline 4-5 months & 8 & 3.8 \\
\hline 6 months & 195 & 92.9 \\
\hline \multicolumn{3}{|l|}{ Complementary feeding } \\
\hline Yes & 246 & 56.4 \\
\hline No & 190 & 43.6 \\
\hline \multicolumn{3}{|c|}{ Starting age of complementary feeding } \\
\hline At 6 months & 190 & 77.2 \\
\hline After 5 months & 22 & 8.9 \\
\hline After 4 months & 20 & 8.1 \\
\hline Since birth & 14 & 5.8 \\
\hline
\end{tabular}

Others* $=$ I do not know 
Most of the respondents, 226(51.8\%) preferred other infant feeding options to EBF. Of these, exclusive replacement feeding (ERF) option accounts for 56(12.8\%) (Figure1).

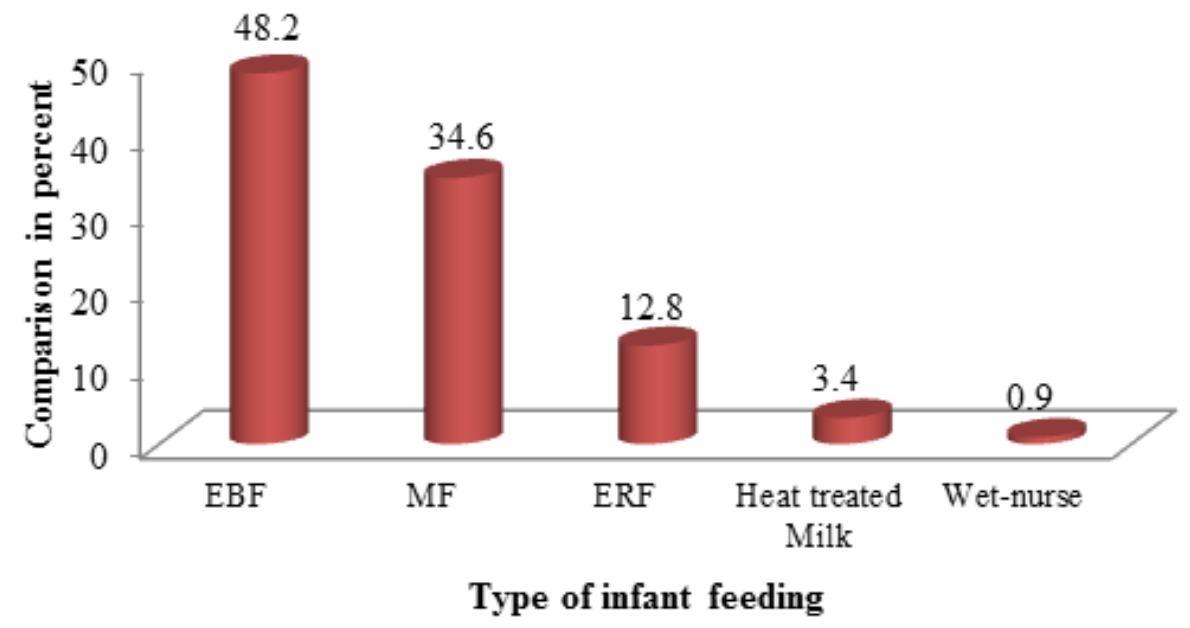

Figure 1. Prevalence of infant feeding options, SNNPR Hospitals, August, 2013.

More than half $(54.9 \%)$ of the respondents choose other infant feeding options because their breast milk is not

sufficient (Figure 2).

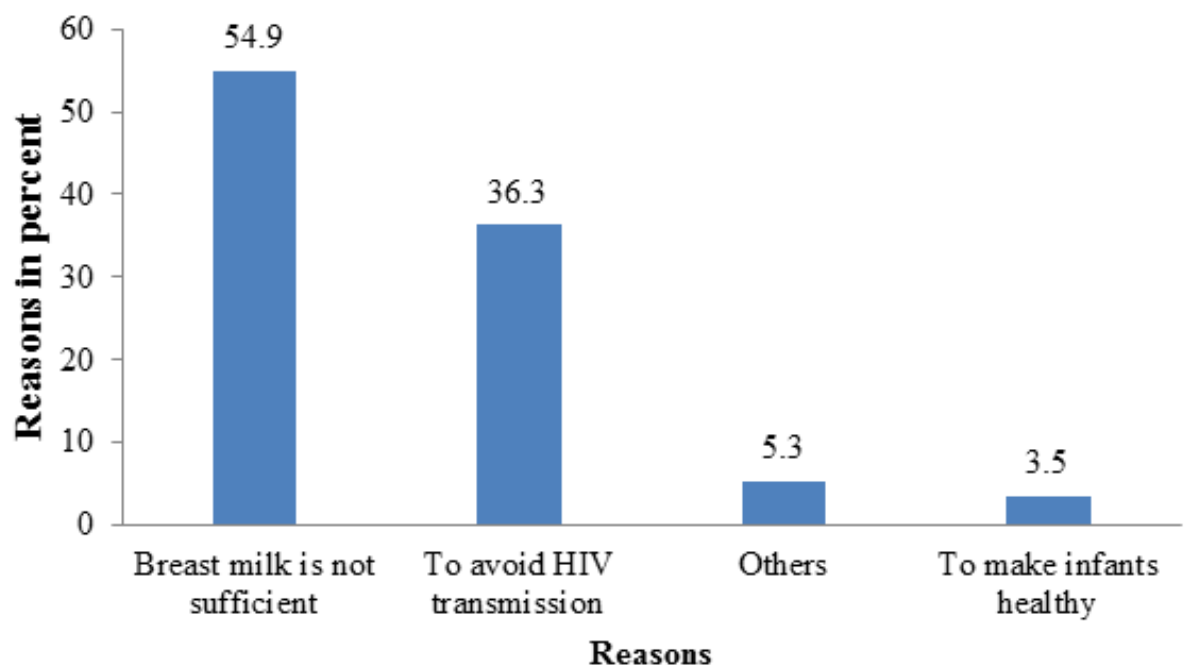

Figure 2. Reasons for choosing other infant feeding options, SNNPR Hospitals, August, 2013

\subsection{Factors Associated with Attitude and Practice of Exclusive Breast Feeding}

Among the respondents, 407(93.3\%) attended ante natal care (ANC) service during pregnancy of last child. Among the respondents who did not attend ANC services, 13(44.8\%) mothers were due to partner imposition 6(20.8\%), 5(17.2\%) and $5(17.2 \%)$ due to too far health facility, mothers had no information about ANC and time scarcity to visit health facility respectively. The finding of this study found out that majority, 383(87.8\%) of the respondents had received counseling on EBF practice. The result of this study also showed, 230(52.8\%) of the respondents had support from their partner on the way of EBF their infant/last child.

Regarding knowledge of mothers on EBF practice, majority 345(79.1\%) had good knowledge about EBF. Of 436 participants, $370(84.9 \%)$ knew the definition of EBF.
Most of the respondents, 383(87.8\%) had knowledge on recommended duration of EBF. Four hundred seven (93.3\%) mothers had heard information on mechanisms of MTCT.

\subsection{Factors Associated with the Attitude of Mothers Towards Exclusive Breast Feeding}

In bivariate and multivariable analysis, some sociodemographic characteristics and selected variables had shown significant association with attitude of mothers towards EBF. Mother's occupation: those mothers who were farmer by occupation were $64 \%$ less likely to have favorable attitude compared to mothers who were housewife ( $\mathrm{AOR}=0.36,95 \% \mathrm{CI}=0.14,0.92)$. In addition, mothers who resided in urban settings of the region were 2 times more likely to have favorable attitude than their counterparts ( $\mathrm{AOR}=2.12,95 \% \mathrm{CI}=1.09,4.20)$.

The finding of this study also showed that, those mother's 
who had good knowledge on EBF practice were almost 3 times more likely to have favorable attitude than their referent group $(\mathrm{AOR}=2.8,95 \% \mathrm{CI}=1.66,4.67)$ and those who were attended ANC service during pregnancy of their last child were 4 times more likely to have favorable attitude than those who did not attend $(\mathrm{AOR}=4.0,95 \% \mathrm{CI}=1.02,16.01)$. Moreover, mothers who received counseling on $\mathrm{EBF}$ practice during ANC visit were 2.6 times more likely to have favorable attitude than those who were not counseled
$(\mathrm{AOR}=2.6,95 \% \mathrm{CI}=1.41,4.89)$.

Multivariable analysis result also indicated that, mothers who disclosed their HIV status to their family were almost 2 times more likely to have favorable attitude towards EBF than the referent group $(\mathrm{AOR}=1.7,95 \% \mathrm{CI}=1.03,2.85)$. In addition, HIV positive mothers who had support from their spouse while EBF their infants were 2 times more likely to have favorable attitude than their counterparts $(\mathrm{AOR}=2.2$, 95\% $\mathrm{CI}=1.43,3.45$ ) (Table 4).

Table 4. Logistic Regression Result for Factors associated with Attitude towards EBF Practice, SNNPR Hospitals, August, 2013.

\begin{tabular}{|c|c|c|c|c|}
\hline \multirow{2}{*}{ Variables } & \multicolumn{2}{|c|}{ Attitude of mothers } & \multicolumn{2}{|l|}{ OR(95\% CI) } \\
\hline & Favorable & Unfavorable & Crude & Adjusted \\
\hline \multicolumn{5}{|l|}{ Occupation of mother } \\
\hline Housewife & $100(61.0 \%)$ & $64(39.0 \%)$ & 1.00 & 1.00 \\
\hline Government employee & $54(63.5 \%)$ & $31(36.5 \%)$ & $1.12(.65,1.92)$ & $0.82(.45,1.49)$ \\
\hline Merchant & $61(55.0 \%)$ & $50(45.0 \%)$ & $0.78(.48,1.27)$ & $0.62(.37,1.05)$ \\
\hline Farmers & $8(29.6 \%)$ & $19(70.4 \%)$ & $0.27(0.11,0.65)$ & $0.36(0.14,0.92)^{*}$ \\
\hline Others* & $24(49.0 \%)$ & $25(51.0 \%)$ & $0.61(.32,1.17)$ & $0.65(.32,1.34)$ \\
\hline \multicolumn{5}{|l|}{ Residence } \\
\hline Rural & $20(29.9 \%)$ & $47(70.1 \%)$ & 1.0 & 1.0 \\
\hline Urban & $227(61.5 \%)$ & $142(38.5 \%)$ & $3.7(2.09,6.47)$ & $2.12(1.09,4.20)^{*}$ \\
\hline \multicolumn{5}{|l|}{ Mother's knowledge } \\
\hline Poor & $29(31.9 \%)$ & $62(68.1 \%)$ & 1.00 & 1.00 \\
\hline Good & $218(63.2 \%)$ & $127(36.8 \%)$ & $3.7(2.24,6.0)$ & $2.8(1.66,4.67)^{*}$ \\
\hline \multicolumn{5}{|l|}{ ANC attendance } \\
\hline No & $4(13.8 \%)$ & $25(86.2 \%)$ & 1.00 & 1.00 \\
\hline Yes & $243(59.7 \%)$ & $164(40.3 \%)$ & $9.3(3.16,27.1)$ & $4.0(1.02,16.01)^{*}$ \\
\hline \multicolumn{5}{|l|}{ Counselling service } \\
\hline No & $18(34.6 \%)$ & $34(65.4 \%)$ & 1.00 & 1.00 \\
\hline Yes & $228(59.5 \%)$ & $155(40.5 \%)$ & $2.8(1.52,5.1)$ & $2.6(1.41,4.89)^{*}$ \\
\hline \multicolumn{5}{|l|}{ HIV disclosure to family } \\
\hline No & $47(39.2 \%)$ & $73(60.8 \%)$ & 1.00 & 1.00 \\
\hline Yes & $199(63.2 \%)$ & $116(36.8 \%)$ & $2.7(1.73,4.11)$ & $1.7(1.03,2.85)^{*}$ \\
\hline \multicolumn{5}{|l|}{ Support from Spouse } \\
\hline No & $89(43.2 \%)$ & $117(56.8 \%)$ & 1.00 & 1.00 \\
\hline Yes & $158(68.7 \%)$ & $72(31.3 \%)$ & $2.9(1.95,4.27)$ & $2.2(1.43,3.45)^{*}$ \\
\hline
\end{tabular}

$*=$ P-Value $<0.05$

\subsection{Factors Associated with Exclusive Breast Feeding Practice}

Socio-demographic characteristics, such as; mother's education had strong association with EBF practice. This is explained as: mother's who completed higher education were 4 times more likely to EBF their infants/last child than those mothers who could not read and write $(A O R=4.3,95 \%$ $\mathrm{CI}=1.29,14.17)$. Father's educational status was also found to be strong independent predictor affecting EBF practice. Those mothers whose spouses were completed higher education were almost 3 times more likely to EBF their infants as compared to those whose spouses could not read \& write $(\mathrm{AOR}=2.87,95 \% \mathrm{CI}=1.00,8.21)$. Mother's whose occupation was daily laborer and unemployed were $63 \%$ less likely to practice EBF than those who were housewife ( $\mathrm{AOR}=0.37,95 \% \mathrm{CI}=0.18,0.75)$.

HIV positive mothers who attended ANC services during pregnancy of last child, were 3.2 times more likely to EBF their infants/last child than those who did not attend ( $\mathrm{AOR}=3.2,95 \% \mathrm{CI}=1.25,8.24)$. Similarly, those mother's who were counseled on EBF while attending ANC were 2.6 times more likely to practice EBF than the referent group $(\mathrm{AOR}=2.57,95 \% \mathrm{CI}=1.22,5.41)$. Furthermore, mother's who had good knowledge on EBF were 2 times more likely to EBF their infants than those who had poor knowledge $(\mathrm{AOR}=1.91,95 \% \mathrm{CI}=1.06,3.45)$. Place of delivery had also shown significance association with EBF practice: Mothers who gave birth at health posts were $84 \%$ less likely to practice EBF than their counter parts $(\mathrm{AOR}=0.16$, $95 \% \mathrm{CI}=0.05,0.56$ ).

On the other hand, mother's who experienced different types of breast illness while EBF their infants were 58\% less likely to EBF their infants than those who did not contract any type of illness $(\mathrm{AOR}=0.42,95 \% \mathrm{CI}=0.24,0.73)$ and those mothers who disclosed their HIV status to their spouse 
were almost 3 times more likely to $\mathrm{EBF}$ their infants/last child (AOR=2.97, 95\%CI=1.38, 6.41) (Table 5).

Table 5. Logistic Regression Result for Factors Affecting EBF Practice, SNNPR Hospitals, August, 2013.

\begin{tabular}{|c|c|c|c|c|}
\hline \multirow{2}{*}{ Predictor variables } & \multicolumn{2}{|c|}{ EBF Practice } & \multicolumn{2}{|l|}{ OR, $95 \%$ CI } \\
\hline & Yes & No & Crude & Adjusted \\
\hline \multicolumn{5}{|l|}{ Mother's education } \\
\hline Cannot read and write & $35(28.2 \%)$ & $89(71.8 \%)$ & 1.00 & 1.00 \\
\hline Only read and write & $41(42.7 \%)$ & $55(57.3 \%)$ & $1.9(1.08,3.33)$ & $1.70(0.87,3.23)$ \\
\hline Primary education & $68(56.2 \%)$ & $53(43.8 \%)$ & $3.26(1.92,5.55)$ & $2.75(1.43,5.27)^{*}$ \\
\hline Second. Education & $43(64.2 \%)$ & $24(35.8 \%)$ & $4.6(2.42,8.59)$ & $2.50(1.19,5.28)^{*}$ \\
\hline Higher education & $23(82.1 \%)$ & $5(17.9 \%)$ & $11.7(4.12,33.20)$ & $4.27(1.29,14.17)^{*}$ \\
\hline \multicolumn{5}{|l|}{ Father's education } \\
\hline Cannot read and write & $10(29.4 \%)$ & $23(70.6 \%)$ & 1.00 & 1.00 \\
\hline Only read and write & $23(26.4 \%)$ & $64(73.6 \%)$ & $0.83(0.34,1.10)$ & $0.62(0.23,1.67)$ \\
\hline Primary education & $43(47.3 \%)$ & $48(52.7 \%)$ & $2.1(.88,4.82)$ & $1.40(0.51,3.60)$ \\
\hline Second. Education & $59(55.1 \%)$ & $49(44.9 \%)$ & $2.8(1.20,6.37)$ & $1.56(0.58,4.18)$ \\
\hline Higher education & $67(69.8 \%)$ & $29(30.2 \%)$ & $5.3(2.25,12.57)$ & $2.87(1.02,8.21)^{*}$ \\
\hline \multicolumn{5}{|l|}{ Occupation of mother } \\
\hline Housewife & $79(48.2 \%)$ & $85(51.8 \%)$ & 1.00 & 1.00 \\
\hline Government employee & $54(63.5 \%)$ & $31(36.5 \%)$ & $1.87(1.09,3.21)$ & $1.63(.93,2.83)$ \\
\hline Merchant & $56(50.5 \%)$ & $55(49.5 \%)$ & $1.1(.68,1.77)$ & $0.97(0.59,1.61)$ \\
\hline Farmers & $7(25.9 \%)$ & $20(74.1 \%)$ & $0.38(.15, .94)$ & $0.53(0.20,1.42))$ \\
\hline Others* & $14(28.6 \%)$ & $35(71.4 \%)$ & $0.43(.22, .86)$ & $0.37(0.18,0.75)^{*}$ \\
\hline \multicolumn{5}{|l|}{ ANC Service } \\
\hline No & $6(20.7 \%)$ & $23(79.3 \%)$ & 1.00 & 1.00 \\
\hline Yes & $204(50.1 \%)$ & $203(49.9 \%)$ & $3.85(1.54,9.66)$ & $3.20(1.25,8.24)^{*}$ \\
\hline \multicolumn{5}{|l|}{ Counselling on EBF } \\
\hline No & $12(23.1 \%)$ & $41(76.9 \%)$ & 1.00 & 1.00 \\
\hline Yes & $198(51.7 \%)$ & $185(48.3 \%)$ & $3.57(1.82,7.01)$ & $2.57(1.22,5.41)^{*}$ \\
\hline \multicolumn{5}{|l|}{ Place of delivery } \\
\hline Hospital & $147(53.6 \%)$ & $127(46.4 \%)$ & 1.0 & 1.0 \\
\hline Health canter & $47(46.1 \%)$ & $55(53.9 \%)$ & $0.74(0.47,1.17)$ & $0.90(0.55,1.45)$ \\
\hline Health post & $3(12.5 \%)$ & $21(87.5 \%)$ & $0.12(0.04,0.42)$ & $0.16(0.05,0.56)^{*}$ \\
\hline Home & $13(36.1 \%)$ & $23(63.9 \%)$ & $0.49(0.24,1.00)$ & $0.88(.39,2.01)$ \\
\hline \multicolumn{5}{|l|}{ Knowledge } \\
\hline Poor & $25(27.5 \%)$ & $66(72.5 \%)$ & 1.00 & 1.00 \\
\hline Good & $185(53.6 \%)$ & $160(46.4 \%)$ & $3.05(1.84,5.07)$ & $1.91(1.06,3.45)^{*}$ \\
\hline \multicolumn{5}{|l|}{ Breast problem } \\
\hline No & $175(51.6 \%)$ & $164(48.4 \%)$ & 1.0 & 1.0 \\
\hline Yes & $35(36.1 \%)$ & $62(63.9 \%)$ & $0.53(.33, .84)$ & $0.42(0.24,0.73)^{*}$ \\
\hline \multicolumn{5}{|l|}{ HIV disclosure to partner } \\
\hline No & $43(72.4 \%)$ & $16(27.6 \%)$ & 1.00 & 1.00 \\
\hline Yes & $183(48.5 \%)$ & $194(51.5 \%)$ & $2.8(1.51,5.12)$ & $2.97(1.38,6.41)^{*}$ \\
\hline
\end{tabular}

Others*: daily laborers and unemployed

* P-Value $<0.05$

\section{Discussion}

\subsection{Attitude Towards Exclusive Breast Feeding}

This study assessed attitude and practice towards EBF and its associated factors among HIV positive mothers attending public hospitals of southern Ethiopia. In this study, one hundred eighty nine (43.3\%) HIV positive mothers had unfavorable attitude towards EBF practice. This finding is lower than the studies conducted in Ivory Cost (62\%) [16], Ibadan Nigeria (96.8\%) [17]. This discrepancy might be due to difference in socio-demographic characteristics of the respondents, difference in data collection procedures and sample size. For instance, in study of Ibadan Nigerian the sample size was larger than our study (513).

This study also found out that, 160 (36.7\%) mothers accepted exclusive replacement feeding/breast milk substitute while only eighty seven $(20.0 \%)$ mothers agreed to breast feed their child by wet-nurse. Similar study done in Ivory Cost showed, $83 \%$ of respondents accepted breast milk substitute, while $(37 \%)$ of them agreed to get their child breastfeed by a wet-nurse [16]. There is a large gap between the two studies, probably because of difference in cultural influence, attitude towards ERF, knowledge about the importance of exclusive replacement feeding and difference in economical potential to afford this type of feeding from the market.

According to the findings of this study, 339(77.8\%) respondents agreed that EBF is the best choice of infant feeding and 364(83.5\%) mothers became happy to EBF their infants. A study done in Tygerberg supported this finding [18]. This may highlight that, most of the mothers clearly understood as; EBF is the single most effective infant's nutrition which does not require any cost, particularly for HIV exposed infants. This study demonstrated that, (82.6\%) mothers agreed that breast milk is nutritionally complete. 
Whereas $(46.8 \%)$ respondents agreed that formula feeding is nutritionally complete. Paradoxically, a study in Tygerberg revealed out that, $(77.8 \%)$ mothers agreed, breast feeding is nutritionally complete, while $(72.2 \%)$ of them agreed formula feeding is nutritionally complete. In our study, the difference between breast feeding and formula feeding was clearly depicted, unlike the finding of a study done in Tygerberg, which showed almost similar proportion in nutritional value of both breastfeeding and formula feeding [18]. This might be due to difference in accuracy of measurement tools during data collection procedure and knowledge gap in study population, about the benefit of breastfeeding.

Mother's occupation had also significant association with attitude towards EBF. This might indicate that, mothers whose occupation were outside their home like farmers may not have time to breast feed their infants and to get adequate information about EBF as compared to housewives. In addition, mothers who resided in urban settings were found to have favorable attitude towards EBF than a rural counterparts. This is possibly because; mothers who are residing in urban settings might have better information on the risks and benefits of breastfeeding in the context of HIV than those who are living in rural areas of the region.

In this study, knowledge of mothers, ANC attendance and counseling service were found to be the major determinant factors affecting attitude of mothers towards EBF. This may indicate that, those mothers who were attending ANC might be counseled and well informed on EBF practice as a routine activity which may result in good knowledge on EBF. This in turn, may positively contribute for change in mother's attitude towards EBF practice.

Most importantly, in the prevention of MTCT, partner/male involvement would play a great role. In line with this, the finding of this study revealed that, mothers who had support from their spouse while EBF their infants were 2 times more likely to have favorable attitude than their counterparts. Besides partner support, disclosing once HIV status to family members may reduce social stigma and fear imposed by HIV. This disclosure may enable them to get strength and full empowerment to have favorable attitude towards EBF practice. The finding of this study also supported this fact in a way that, those mothers who disclosed their HIV status to their family were almost 2 times more likely to have favorable attitude towards EBF than those who did not disclose their HIV status.

\subsection{Exclusive Breast Feeding Practice}

In this study, nearly half (48.2\%) of the mothers practiced EBF. This finding is in lined with the study done in Bahir Dar town $(50.2 \%)$ [19].This finding is slightly greater and not consistent with studies conducted in China (4.2\%) [20], India (30\%) [21], Ibadan (28.3\%) [17], South Africa (35.6\%) [22], Nigeria (46\%) [23], Zambia (35\%) [24] and Uganda (31.5\%) [25] Nonetheless, this finding is comparatively lower than the findings of studies done in Tanzania (78.7\%) [10] and Sokoto Nigeria (58\%) [26]. The discrepancy of these findings might be due to difference in culture of feeding habits, study time, difference in economic potential, different health policy and strategies of interventions.

The result of this study also revealed that, the proportion of mothers practicing EBF (48.2\%) was comparatively higher than that was found in our country: Addis Ababa (30.6\%) [27] and Guraghe zone (16.2\%) [15]. The differences between these findings might be due to the fact that the time at which these studies conducted was different from our study. For instance; study done in Guraghe zone was in 2004 and Addis Ababa in 2008. This might indicate that as time goes on there is a change in policy, strategy and improvement in implementation of the program. However, the proportion of mothers practicing EBF was significantly lower than what was observed in the finding of study in Gondar (83.7\%) [4]. This might be due to difference in characteristics of study population and the study area.

Colostrums/first milk which has high concentration of nutrients is very mandatory for optimal growth and development of newborn. On the basis of this fact, this study revealed out that, $(51.5 \%)$ mothers put their baby to their breast immediately after birth, $(28.5 \%)$ within 1 hour and $(17.2 \%)$ within 30 minutes after birth. In this study, the proportion of mothers who initiated their breast within 1 hour after delivery was found to be higher than the study carried out in Sokoto Nigeria (8.0\%) [26] and much lower than the finding of study in Ibadan (50.8\%) [17]. This is probably due to the cultural habit and the chance to afford replacement feeding, so as to avoid breastfeeding. In this study, (8.7\%) mothers were offered fluids and solid foods (pre-lacteal feeding) to their infants/last child immediately after birth before breast feeding. Among reasons for providing prelacteal feeding were: milk did not come in yet $(63.2 \%)$, mothers were ill (18.4\%) and babies were ill (7.9\%). Similar study in eastern Uganda showed inconsistent result where (64\%) mothers provided pre-lacteal feeding for their infants [28]. The possible reasons might be knowledge gap on the benefits of EBF in reducing post natal HIV transmission and difference in PMTCT program implementation among study areas.

On this finding, of 210(48.2\%) EBF mothers, (86.7\%) of them were EBF for 6months. Although the highest proportions of those mothers EBF for 6 months, the rest of the respondents were not properly breastfed their infants/last child as per the WHO recommendation of EBF duration. This might be due to fear of HIV transmission, social stigma, pressure from others and inconsistence in attending HIV prevention program. This finding is not similar with the study done in Tanzania $(80.1 \%)$, where a higher proportion of mothers EBF for 6 months [1]. The discrepancy might be due to variation in sample size and study population. The result of this study also indicated that $48.2 \%, 34.6 \%$ and $12.8 \%$ mothers EBF, mixed fed and formula fed their infants respectively. This finding is differed from other studies [17, 22, 24-26]. This is probably due to improvement in program implementation and health seeking behavior of the community. As it has been recommended by WHO and 
UNICEF EBF is practiced for 6 months of life followed by complementary feeding since then. This study found out that, of 246 mothers practicing complementary feeding, $77.2 \%$ mothers started at the right time/ 6 months of life. This finding had discrepancy with the study done in Cotd'Ivoire where $77 \%$ mothers introduced complementary foods to their infants within the seventh month of life [29]. The difference might be due to culture of feeding and socio-economical variation.

In multivariable analysis, the finding of this study demonstrated that, HIV positive mothers who were completed higher education showed strong association with EBF practice. This finding is in lined with the study done in eastern Uganda [29]. This could be explained as; mothers who are well educated may have better understanding and the ability to easily identify the risks and benefits of breastfeeding. In addition, those mothers whose occupation was daily laborer and unemployed were $63 \%$ less likely to practice EBF than those who were housewife by occupation. This might be due to the fact that those mothers may not have time to EBF their infant in search of jobs and stay outside for many hours to fulfill their subsistence life that may result in exhaustion. Whereas, those mothers who were housewife may have an ample of time to provide care and EBF their infants.

Furthermore, mothers who delivered at health post were $84 \%$ less likely to practice EBF than those mothers who delivered at hospital. This finding is highly consistent with the finding of study conducted in Tanzania [10]. This may be because of repeated exposure to qualified health workers while providing ANC services, counseling on infant feedings, regular assessment and follow up for ART medication and better health services provision available at hospital as compared to health post. In the present study, mother's knowledge on EBF, ANC attendance and counseling had significant association with EBF practice. This finding is comparable with the finding of study done in Tanzania [10] and India [30].

On the other hand, mother's breast problem was found to be strong independent predictor affecting EBF practice. The report from Tanzania also supported this finding [10]. According to this study, mothers who disclosed their HIV status to their spouses were 3 times more likely to EBF their infants, which is consistent with finding of Gondar study [4]. This is probably due to the fact that, stigma and discrimination of HIV infected mothers may hinder them to EBF their infants. Thus, if they are in a position to disclose themselves to their partner/spouse, they might have better opportunity to practice EBF without any fear and their ability to make their own decision may also increase.

\section{Strength and Limitations of the Study}

\subsection{Strength}

This study tried to address the attitude and practice towards exclusive breast feeding and its associated factors among HIV positive mothers.

\subsection{Limitation}

Since the age gap of infants was 2 years; social desirability bias are the potential limitations of this study. However, numerous scientific procedures have been employed to minimize the possible effects. To reduce the response bias, for instance, the aim of the study was discussed with respondents in order to obtain genuine response. In addition, procedures such as supervision, pretest of data collection tool, and adequate training of data collectors and supervisors were utilized. This study also not supplemented with qualitative method of data collection.

\section{Conclusion and Recommendation}

In this study, the attitudes of mothers towards EBF and EBF practice were still very low. On the other hand, significant proportion of mothers practiced mixed feeding as first infant feeding methods for fear of HIV transmission and breast milk insufficiency. This finding also revealed out that, mothers of better educational level and housewife by occupation were found to have more opportunity to EBF their infants than their counterparts.

In addition, ANC attendance, counseling on safer infant feeding option, place of delivery and knowledge on EBF had significant association with attitude and EBF practice. Furthermore, partner support on the way of EBF infants, HIV disclosure to partner as well as family members were other factors affecting attitude and EBF practice of HIV positive mothers. Concerted efforts need to be exerted by police makers and stake holders in designing further strategies and strengthening the system.

\section{List of Abbreviations}

ANC: Ante Natal Care, AIDS: Acquired Immune Deficiency Syndrome, ART: Anti-Retroviral Therapy, EBF: Exclusive Breast Feeding, HIV: Human Immune Virus, MTCT: Mother To Child Transmission, PMTCT: Prevention Of Mothers To Child Transmission, SNNPRS:

Southern Nation Nationalities and People's Regional State, UNICEF: United Nations Children's Emergency Fund, WHO: World Health Organization

\section{Authors' Contributions}

KEM participated in the design of the study, data collection, analyzed the data and drafted the paper. NWA participated in the analysis and revised subsequent drafts of the paper. Both authors read and approved the final manuscript.

\section{Acknowledgements}

We would like to thank University of Gondar for their financial support. We are also grateful to SNNPRS Health 
Bureau for their guidance and support during data collection process.

\section{References}

[1] Young SL, Israel- Ballard KA, Dantzer EA, Ngonyani MM, Nyambo M,Ash DM, Chantry CJ: Infant feeding practices among HIV-positive women in Dares Salaam, Tanzania, indicate a need for more intensive infant feeding counseling. Public Health Nutrition 2010, 13(12):2027-2033.

[2] Ogundele MO, Coulter JB: HIV transmission through breastfeeding: problems and prevention. Ann Trop. Paediatr 2003, 23(2):91-106.

[3] FHAPCO/FMOH: Guidelines for Pediatrics HIV/AIDS care and Treatment in Ethiopia: FHAPCO/FMOH; 2008.

[4] Muluye D, Woldeyohannes D, Gizachew M, Tiruneh M: Infant feeding practice and associated factors of HIV positive mothers attending prevention of mother to child transmission and antiretroviral therapy clinics in Gondar Town health institutions. BMC public health 2012, 12: 240.

[5] Federal Ministry of Health: National strategy for Infant and Young Child Feeding (IYCF). Ethiopia: Federal Ministry of Health, Family Health Department; 2004.

[6] Fletcher FE, Ndebele P, Kelley MC: Infant feeding and HIV in Sub-Saharan Africa, what lies beneath the dilemma?. Theor Med Bioeth 2008, 29 (5):307-30.

[7] Introduction to HIV and breastfeeding. http://www.avert.org/hiv-andbreastfeeding.htm(07/03/2015).

[8] U.S. Department of Health and Human Services, Office on Women's Health. Your Guide to breast feeding, 2011.

[9] Doherty T, Sanders D, Goga A, Jackson D: Implications of the new WHO guidelines on HIV and infant feeding for child survival in South Africa. Bulletin of the World Health Organization 2011, 89(1):62-67.

[10] Nkala TE, Msuya SE: Prevalence and predictors of EBF among HIV positive women in Kigoma region, Western Tanzania. Int. Breastfeed J 2011,6(1):17

[11] Pool R, Nyanzi S, Whitworth JA: Breastfeeding practices and attitudes relevant to the vertical transmission of HIV in rural south-west Uganda. Ann.Trop.Paediatr 2001,21(2):119-25.

[12] Dop MC: Breastfeeding in Africa: will positive trends be challenged by the AIDS epidemic. Santé 2002, 12(1):64-72.

[13] Nakunda J, Tumwine J, Nankabiwa V, Tylleskar T: "She would sit with me" mothers experience, of individual peer support for exclusive breastfeeding in Uganda. Int Breastfeeding Journal 2010, 5(16):1-13.

[14] Federal Ministry of Health, Ethiopian Health and Nutrition Research Institute: Report on the 2007 Round ANC Sentinel HIV surveillance in Ethiopia. Addis Ababa: Federal Ministry of Health, Ethiopian Health and Nutrition Research Institute; 2010 .

[15] Belachew T, Jira C: Awareness about feeding options for infants born to HIV positive mothers and mother to child transmission of HIV in Gurage zone, South Ethiopia. Ethiop. J.
Health Dev 2007, 21(1):40-47.

[16] Yeo EA, Béquet L, Ekouévi DK, Krawinkel M: Attitudes towards exclusive breastfeeding and other infant feeding options--a study from Abidjan, Cote d'Ivoire. J Trop Pediatr 2005, 51(4):223-6.

[17] Brown BJ. Oldakun RE, Osinusi K: Situation analysis of the existing infant feeding pattern at the commencement of the prevention of mother to child transmission (PMTCT) of HIV programme in Ibadan. Nigerian journal of clinical practice 2009, 12(4):421-8.

[18] Petri K. Schmidt D. Schwarz E: Knowledge, attitudes and practices of women regarding the prevention of mother-tochild transmission (PMTCT) programme at the Vanguard Community Health Centre, Tygerberg. SAJCN 2007, 20(2):71.

[19] Seid AM, Yesuf ME, Koye DN: Prevalence of Exclusive Breastfeeding Practices and associated factors among mothers in Bahir Dar city, Northwest Ethiopia: a community based cross-sectional study. Int Breastfeed J 2013, 8: 14.

[20] Shi L, Zhang J, Wang Y, Guyer B: Breast feeding in rural China: Association between knowledge, attitudes, and practices. J Hum. Lact 2008, 24(4):377-85

[21] Phadke MA, Gadgil B, Bharucha KE, Shrotri AN, Sastry J, Gupte NA, Brookmeyer R, Paranjape RS, Bulakh PM, Pisal H, Suryavanshi N, Shankar AV, Propper L, Joshi PL Bollinger RC: Replacement-fed infants born to HIV-infected mothers in India has a high early postpartum rate of hospitalization. Journal Nutr 2003, 133(10):3153-7.

[22] Ladizani R, Peltzer K, Motlatso G.Mlambo, Phaweni K: Infant-feeding practices and associated factors of HIV-positive mothers at Gert Sibande South Africa. Human Science research council of South Africa 2010,11(11):1651-2227.

[23] Mohammed A, Shehu A, Aliyu A, Zoaka A: Infant feeding options, Practices and determinants of feeding practice among HIV positive mothers in Abuja, Nigeria. Niger Med. J 2010, 51(1): 14-17.

[24] Omari AA, Luo C, Kankasa C, Bhat GJ, Bunn J: Infantfeeding practices of mothers of known HIV status in Lusaka, Zambia. Health Policy Plan 2003, 18(2):156-62.

[25] Babirye J N, Nuwaha F, Grulich A E: Adherence to feeding guidelines among HIV-infected and HIV uninfected mothers in a rural district in Uganda. East African medical journal 2009, 86(7):337-43.

[26] Oche MO, Umar AS: Breastfeeding practices of mothers in a rural community of Sokoto, Nigeria. Niger Postgrad Med J 2008, 15 (2):101-4.

[27] Maru Y, Haidar J: Infant feeding practice of HIV positive mothers and its determinants in selected health institutions of Addis Ababa, Ethiopia. Ethiop. J. Health Dev 2009,23 (2):107-114

[28] Ingunn MS Engebretsen, Wamani H, Karamagi C, Semiyaga $\mathrm{N}$, Tumwine J, Tylleskar T: Low adherence to exclusive breastfeeding in Eastern Uganda: A community- based crosssectional study. BMC Pediatrics 2007, 7:10

[29] Becquet R, Leroy V, Ekouevi DK: Complementary feeding adequacy in relation to nutritional status among early weaned breastfed children who are born to HIV-infected mothers: Abidjan, Cote d'Ivoire. Pediatrics 2006, 117(4):701-10. 
[30] Kishore MS, Kumar P, Aggarwal AK: Breastfeeding knowledge and practices amongst mothers in North India. J Trop Pediatr 2009, 55(3):183-8 